\title{
Electrophysiological monitoring during preoperative angiography to guide decisions regarding permanent occlusion of major radicular arteries in patients undergoing total en bloc spondylectomy
}

\author{
Khalil Salame, MD, ${ }^{1,3}$ Shimon Maimon, MD, ${ }^{2}$ Gilad J. Regev, MD,,3 Tali Jonas Kimchi, MD, ${ }^{2}$ \\ Akiva Korn, MMedSc, ${ }^{1}$ Laurence Mangel, PhD, ${ }^{1}$ and Zvi Lidar, MD ${ }^{1,3}$ \\ 'Department of Neurosurgery, The Spine Unit, Tel Aviv Medical Center; ${ }^{2}$ Department of Radiology, Tel Aviv Medical Center; and \\ ${ }^{3}$ Sackler Faculty of Medicine, Tel Aviv University, Tel Aviv, Israel
}

\begin{abstract}
OBJECTIVE Preoperative embolization is performed before spine tumor surgery when significant intraoperative hemorrhage is anticipated. Occlusion of radicular and segmental arteries may result in spinal ischemia. The goal of this study was to check whether neurophysiological monitoring during preoperative angiography in patients scheduled for total en bloc spondylectomy (TES) of spine tumors improves the safety of vessel occlusion.
\end{abstract}

METHODS This was a case series study of patients who underwent tumor embolization under somatosensory evoked potential (SSEP) and motor evoked potential (MEP) monitoring in preparation for TES in treating spine tumors. The angiography findings, the embolized vessels, and the results are presented.

RESULTS Five patients whose ages ranged from 33 to 75 years and who had thoracic spine tumors are reported. Four patients suffered from primary tumor and 1 patient had a metastatic tumor. Radicular arteries at the tumor level, 1 level above, and 1 level below were permanently occluded when SSEPs and MEPs were preserved during temporary occlusion. No complications were encountered during or after the angiography procedure and embolization.

CONCLUSIONS Temporary occlusion with electrophysiological monitoring during preoperative angiography may improve the safety of permanent radicular artery occlusion, including the artery of Adamkiewicz in patients undergoing TES for the treatment of spine tumors.

http://thejns.org/doi/abs/10.3171/2016.5.FOCUS16140

KEY WORDS spine tumors; total en bloc spondylectomy; preoperative embolization; electrophysiological monitoring

$\mathrm{T}$ OTAL en bloc spondylectomy (TES) is a radical surgical procedure indicated for the treatment of selected patients with primary malignant spine tumors, aggressive benign primary osseous tumors, and solitary metastatic tumors in individuals with no other metastases and with a relatively long life expectancy. During the last 2 decades this technique has gained recognition among spine surgeons as the preferred method of treatment for selected patients with definite indications. Several studies have shown that TES resulted in lower tumor recurrence and improved survival compared with intralesional resection. ${ }^{1,5,9,11,22,23,28}$ Yet, TES is an aggressive procedure that requires extensive dissection of the spine and adjacent structures, with potentially serious complications. A systematic review by the Spine Oncology Study
Group showed that en bloc resection of spine tumors is achievable, but the adverse event profile is high even at experienced centers ${ }^{27}$ One of the major risks of this operation is massive intraoperative bleeding, which may result from tumor tissue, epidural veins, adjacent bones, or from injury to major blood vessels.

Different vascular complications related to spine surgery may be encountered in anterior approaches other than TES. Oskouian and Johnson ${ }^{20}$ reported their experience with vascular complications in anterior thoracolumbar reconstruction, revealing that the risk of intraoperative bleeding, although rare, is still a real concern. Intraoperative hemorrhage is a significant risk in spine tumor surgery also when corpectomy is performed via posterior approaches. Bleeding may impair visibility, hinder tumor

ABBREVIATIONS AKA = artery of Adamkiewicz; ASA, PSA = anterior, posterior spinal artery; EPM = electrophysiological monitoring; MEP = motor evoked potential; RMA = radiculomedullary artery; SSEP = somatosensory evoked potential; TES = total en bloc spondylectomy.

SUBMITTED March 31, 2016. ACCEPTED May 23, 2016.

INCLUDE WHEN CITING DOI: 10.3171/2016.5.FOCUS16140. 
resection, and increase the operative time. Preoperative embolization is usually recommended in surgery for tumors with high vascularity. ${ }^{2,8,12,21,25}$ The hazard of massive intraoperative hemorrhage is more significant in TES, where blood loss may be life threatening; hence blood transfusion is required in almost every case.

Preoperative embolization and/or intraoperative ligation of the radicular arteries is used to reduce the risk of bleeding during surgery. Some authors have proposed closure of the radicular arteries at the 3 vertebral levels encompassing the tumor. ${ }^{13,17,25}$ However, the safety of this occlusion is still controversial, especially in the midthoracic spine, where previous studies have reported acute and severe neurological deficits as a result of sacrifice or inadvertent injury of segmental arteries during spine or vascular surgical procedures or preoperative embolization. ${ }^{15,18,19}$ Matsuda and colleagues ${ }^{15,16}$ reported that the incidence of paraplegia, temporary or permanent, in patients undergoing thoracic endovascular aneurysm repair with closure of the intercostal-lumbar artery that supplies the artery of Adamkiewicz (AKA; also called the arteria radicularis magna) was significantly high.

The goal of the present case series study was to check whether the use of electrophysiological monitoring (EPM) during temporary occlusion of radicular or radiculomedullary arteries (RMAs) while performing preoperative angiography can predict the safety of occluding these vessels in patients scheduled for TES of thoracic spine tumors.

\section{Methods \\ Patient Characteristics}

This study was approved by our local institutional review board. Five patients who underwent preoperative embolization under EPM prior to TES for tumors of the thoracolumbar spine were included in the study. These patients' characteristics are presented in Table 1. All patients were initially subjected to CT-guided needle biopsy for histopathological diagnosis of their tumors before deciding on the definitive surgical procedure. All patients underwent preoperative angiography and EPM under general anesthesia, with the intent to occlude tumor-feeding vessels as well as radicular arteries at the involved levels, at 1 level above, and at 1 level below the tumor.

\section{The Angiography Procedure}

Angiography was performed with the patients under general endotracheal anesthesia, with somatosensory evoked potential (SSEP) and motor evoked potential (MEP) monitoring. A vascular sleeve was introduced into the common femoral artery and a Cobra catheter was advanced through the sleeve into the target segmental arteries. Contrast material was then injected to obtain selective angiographic studies of the segmental arteries, while suspending ventilation, at 3 levels: the lesion level, 1 level above, and 1 level below. Segmental arteries at the lesion level and adjacent levels that do not contribute to the pial arterial system (i.e., the posterior spinal artery [PSA] and anterior spinal artery [ASA]), were permanently occluded. If the segmental arteries contributed to the AKA or other major RMAs, we then looked for collateral flow from the
TABLE 1. Data in 5 patients who underwent TES

\begin{tabular}{cclc}
\hline Case No. & Sex/Age $(\mathrm{yrs})$ & \multicolumn{1}{c}{ Tumor Type } & Vertebral Level \\
\hline 1 & $\mathrm{~F} / 36$ & Chondrosarcoma & $\mathrm{T}-11$ \\
\hline 2 & $\mathrm{M} / 33$ & Giant cell tumor & $\mathrm{T}-10$ \\
\hline 3 & $\mathrm{M} / 34$ & $\begin{array}{c}\text { Metastatic parotid car- } \\
\text { cinoma }\end{array}$ & $\mathrm{T}-6$ \\
\hline 4 & $\mathrm{M} / 33$ & Osteogenic sarcoma & $\mathrm{T}-11$ \\
\hline 5 & $\mathrm{M} / 75$ & Malignant schwannoma & $\mathrm{T}-10$ \\
\hline
\end{tabular}

posterior RMAs at the target level or from RMAs at other levels. If the collateral arteries were patent, then temporary balloon occlusion of the segmental arteries was done under EPM. If EPM recordings remained unchanged from baseline after 10 minutes of temporary occlusion, and the pial vessels were sufficiently filled from other levels, then the artery was permanently occluded with Onyx, coils, or a combination of both. If significant reduction of the amplitude or a shift in the latency of the evoked potentials was noticed, the temporary occlusion was released and the artery was left patent.

\section{Neurophysiological Intraoperative Monitoring}

We used the NIM Eclipse Spinal System from Medtronic. Neurophysiological data were recorded with a 16/32-channel intraoperative evoked response unit. The SSEPs were collected from subdermal needle electrodes placed at scalp positions $\mathrm{C} 3, \mathrm{C} 4$, and $\mathrm{Cz}$, referenced to $\mathrm{Fpz}$ (according to the International 10-20 scalp positioning system). Bipolar stimulation of either posterior tibial nerve was performed using pregelled surface electrodes placed along the medial malleoli. A ground electrode was placed at the shoulder. Averages of 100-500 sweeps (scale: 100msec window, $0.5-$ to $2-\mu \mathrm{V}$ vertical scale/division) were collected at 4.2 stimulations/second. Criteria for alert included the sudden onset of amplitude attenuation (beyond $30 \%$ ) of the cortical P37 waveform and/or a sudden-onset latency shift of greater than $10 \%$.

Transcranial-electric MEPs were recorded with paired subdermal needle electrodes from the bilateral thenar, anterior tibialis, and abductor hallucis muscles (recording parameters: $100-\mathrm{msec}$ single sweep, $30-$ to $1000-\mu \mathrm{V}$ vertical scale) following transcranial stimulation $(333 \mathrm{~Hz}$, 5-pulse train, 80-190 mA) with subdermal corkscrewstyle electrodes placed $1 \mathrm{~cm}$ anterior to the $\mathrm{C} 3$ and $\mathrm{C} 4$ scalp positions according to the International 10-20 scalp positioning system.

The status of the evoked potential data, including significant changes as well as stability, was brought to the attention of the surgical team throughout the endovascular procedure.

\section{Results}

This case series included 5 patients -4 men and 1 woman-whose ages ranged from 33 to 75 years. Four patients suffered from primary spine tumors and 1 patient from a slow-growing metastatic parotid carcinoma (Table 1).

Overall, the AKA was permanently occluded in 3 
TABLE 2. Angiography procedures and data in 5 patients who underwent TES

\begin{tabular}{cccllll}
\hline $\begin{array}{c}\text { Case } \\
\text { No. }\end{array}$ & $\begin{array}{c}\text { Tumor } \\
\text { Level }\end{array}$ & $\begin{array}{c}\text { AKA } \\
\text { Level }\end{array}$ & $\begin{array}{c}\text { Other Prominent } \\
\text { RMA Levels }\end{array}$ & $\begin{array}{c}\text { Levels of Arteries } \\
\text { Temporarily Occluded }\end{array}$ & $\begin{array}{c}\text { EPM } \\
\text { Changes }\end{array}$ & $\begin{array}{c}\text { Levels of Segmental Arteries } \\
\text { Permanently Occluded }\end{array}$ \\
\hline 1 & T-11 & Rt T-11 & Rt T-10, It T-11, It L-1 & T-10, T-11, T-12 & None & T-10, T-11, T-12 \\
\hline 2 & T-10 & Lt T-11 & Lt T-12 & T-9, T-10, T-11 & None & T-9, T-10, T-11 \\
\hline 3 & T-6 & Rt T-9 & None & T-5, T-6, T-7 & None & T-5, T-6, T-7 \\
\hline 4 & T9-11 & Lt L-1 & None & T-8, T-9, T-10, T-11 & None & T-8, T-9, T-10, T-11 \\
\hline 5 & T-10 & Lt T-11 & Rt T-11, bilat T-12 & T-9, T-10, T-11 & None & T-9, T-10, T-11 \\
\hline
\end{tabular}

cases. Three segmental arteries were permanently closed bilaterally in 4 cases, and 4 segmental arteries were permanently closed bilaterally in 1 patient (Table 2). No EPM changes were recorded in any of the cases during temporary balloon occlusion or after permanent occlusion with Onyx or coils. Control angiography after arterial closure demonstrated adequate filling of the ASA in all cases.

The angiography, including permanent radicular arterial occlusion, was well tolerated by the patients. The estimated blood loss during surgery ranged from 970 to 2280 $\mathrm{ml}$ (mean $1355 \mathrm{ml}$ ). Neither early nor late complications due to embolization were encountered.

\section{Illustrative Cases \\ Case 1}

A 36-year-old woman presented with axial back pain. The results of neurological examination were normal. Spine MRI studies showed a tumor located at the T-11 level involving the vertebral body and the pedicle.

A CT-guided needle biopsy was done, and histopathological findings were consistent with chondrosarcoma (Fig. 1A). On angiography the AKA was demonstrated at the T-11 level on the right side (Fig. 1B). Three additional prominent RMAs were identified at the left T-11, right T-10, and left L-1. Following temporary bilateral occlusion at the T-11 level (Fig. 1C), selective dye injection to the left L-1 showed good retrograde flow to the ASA. Subsequently, when EPM was unchanged after 10 minutes of occlusion, permanent occlusion of the intercostal arteries was performed, including the orifice of AKA (Fig. 1D). The patient tolerated the procedure well and no complications were noted.

Surgery was performed on the next day with TES of T-11 and fusion from T-8 to L-2 via a posterior-only approach. The surgery and the immediate postoperative course were uneventful, and the patient was discharged 10 days after surgery. Six months after surgery she was admitted with fever and axial back pain. An MRI study showed deep wound infection. Wound revision was performed, leaving the hardware in place. She received intravenous antibiotic treatment and made a complete recovery.

Approximately 55 months after the first surgery she presented with back pain, and CT scans showed breakage of the fixation rods, whereas the intervertebral cage seemed to remain in place. During revision surgery the broken rods were replaced by double carbon rods reinforced by 2 cross-connectors and additional bone autograft harvested from the ribs. Five years after surgery the patient is asymptomatic, with no evidence of disease recurrence.

\section{Case 5}

The patient was a 75-year-old man with back pain and spastic paraparesis, and the tumor was located at the T-10 level. A CT-guided needle biopsy was done and histopathological findings were consistent with malignant peripheral nerve sheath tumor. On angiography, the AKA was demonstrated at the T-11 on the left side, with additional prominent RMAs at the T-11 level on the right and at T-12 bilaterally (Fig. 2A). Temporary occlusion of the T-11 radicular arteries bilaterally did not affect the EPM (Fig. 2B). Good filling of the ASA through collateral arteries originating at the T-12 level was observed bilaterally. Hence, permanent occlusion was performed at the T-9, T-10, and T-11 levels without undue complications (Fig. 2C).

Thirty-six hours later, TES of the T-9, T-10, and T-11 vertebrae with the proximal parts of the ribs bilaterally, followed by circumferential fusion from T-7 to L-1, was performed. A dural tear was noticed and repaired using Prolene sutures. Postoperatively, the patient's neurological symptoms improved; however, he developed a pneumonia that resolved with antibiotic treatment and chest physiotherapy. Three weeks after surgery, he was transferred to the oncology department for further treatment.

Approximately 5 months later, he was readmitted with wound dehiscence in the back and underwent operation by a plastic surgeon. However, in the postoperative period he suffered from superior vena cava thrombosis, which was managed conservatively, but he developed respiratory and renal failure and died.

\section{Discussion}

Thirty-one pairs of radicular arteries enter the spinal canal through the intervertebral foramina, but most of these arteries terminate without providing blood supply to the spinal cord. The 3 spinal cord arteries, 1 anterior and 2 posterior, do not receive tributaries at each spinal level; normally fewer than 8 radicular arteries contribute to the spinal cord vessels over its entire length., ${ }^{7,24}$ It is essential to know whether the segmental arteries at the targeted surgical level provide feeders to the ASA. Hence the importance of preoperative angiography with temporary occlusion of any vessels that need to be sacrificed in the process of total en bloc resection of spine tumor.

Dommisse ${ }^{7}$ defined the midthoracic spine as the critical zone for spinal cord injury due to a combination of 2 factors: it has the least abundant blood supply and the narrowest bony boundaries. Anatomical studies have shown that the AKA is usually located between the T- 8 and L-3 levels on the left side. ${ }^{2}$ Other studies have also demon- 

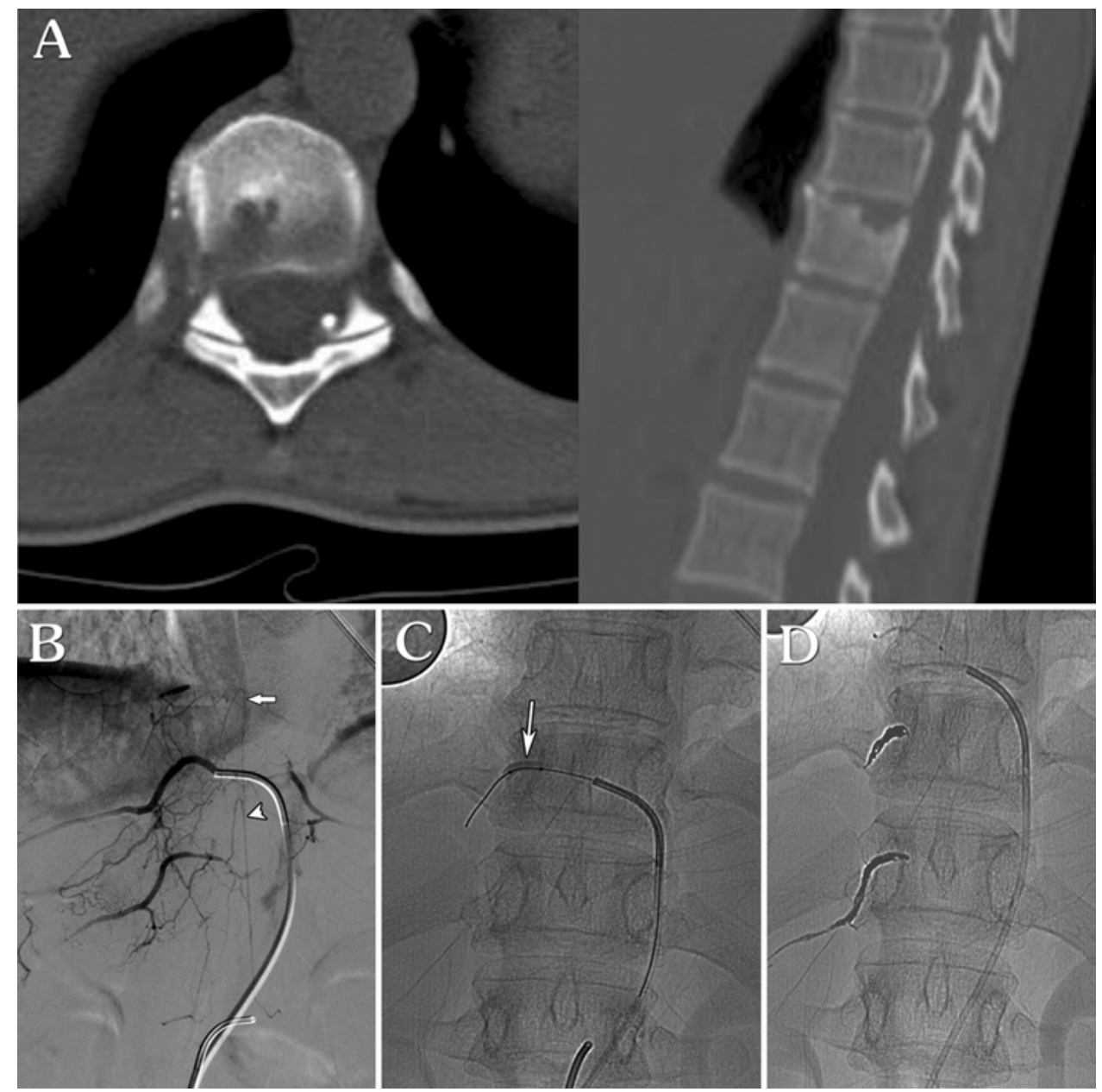

FIG. 1. Case 1. CT and angiography data. A: Axial and sagittal images of the bone window CT demonstrate chondrosarcoma of the upper body and end plate of T-11 vertebra. The embolization procedure is depicted in panels B-D. B: Subtracted angiography of the right T-10 intercostal artery shows opacification of the posterior RMA (arrow). Opacification of the right T-11 intercostal artery shows the RMA (arrowhead). C: Balloon occlusion test. The balloon is inflated in the T-11 intercostal (segmental) artery (arrow). D: Embolization is complete. Coils are placed into the right T-11 and T-12 intercostal arteries.

strated the dominance of the left radicular vascular system over the right. ${ }^{10}$

The TES, which comprises complete removal of the tumor and the involved vertebra in 1 unit, entails the risk of significant intraoperative bleeding. Anterior approaches to the thoracolumbar spine have the risk of direct vascular injury. ${ }^{4,8,12,20,26}$ In the posterior approach there is also a risk for vessel injury, and in this position control over these vessels is not possible. To minimize intraoperative bleeding during this major operation, Kawahara et al. suggested that the surgeon commence with embolization of the radicular arteries at 3 levels bilaterally: at the tumor level, at 1 level above it, and at 1 level below it. ${ }^{13}$ This group performed a study in dogs to check what impact interruption of bilateral segmental arteries in up to 3 levels had on spinal cord blood flow and function. They found that although bilateral occlusion at 3 vertebral levels led to a $75 \%$ reduction in spinal cord blood flow, no neurological impairments ensued. ${ }^{25}$

Other groups reported that during TES, the AKA and other radicular arteries may be sacrificed without causing neurological deficits. Boriani et al.'s group demonstrated retrospectively that no neurological deficits developed in 4 patients in whom the AKA was sacrificed at the level of the intervertebral foramen. ${ }^{3}$ They also identified a new feeding artery on postoperative angiography in all 4 cases, which might explain the sparing of neurological function.

Murakami et al., in their retrospective review of 180 cases of TES, found 15 patients in whom the AKA was located at the involved vertebra. ${ }^{17}$ The artery was occluded during preoperative angiography or during surgery as part of a 3-level occlusion of radicular arteries that was done routinely. They encountered no cases of neurological deterioration.

However, injury of major radicular spinal vessels resulting in paraplegia has long been considered a dreadful complication of spinal, cardiothoracic, and abdominal surgeries as well as of aortic endovascular procedures..$^{411,15,18,19}$ Devastating neurological deficits have been reported even after unilateral ligation of segmental vessels. Orchowski et al. reported 2 cases in which ASA syndrome developed after unilateral ligature of left segmental vessels in the course of anterior thoracic spinal surgery. ${ }^{19}$ 

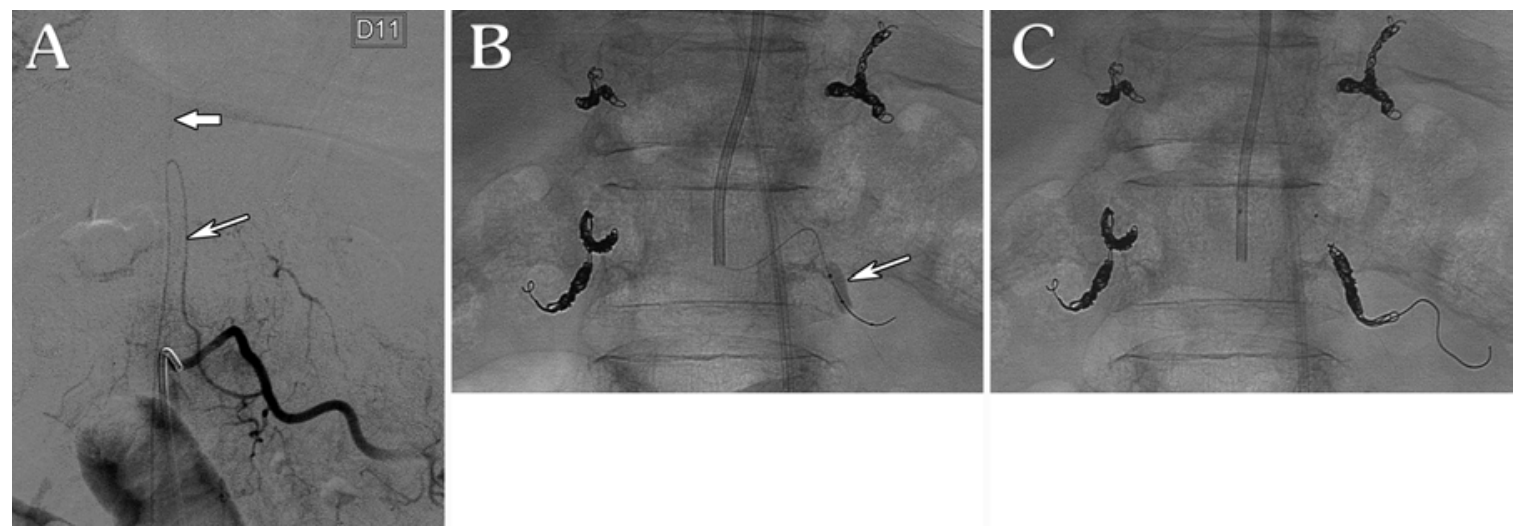

FIG. 2. Case 5. Angiography data. A: Subtraction angiography of the left T-11 intercostal artery shows the RMA (thin arrow) supplying the ASA (thick arrow). B: During the occlusion test the balloon is inflated in the T-11 intercostal artery (arrow). C: Coils are placed in the left T-11 intercostal artery after the occlusion test. (Coiling was already done in the other intercostal arteries.)

In a large series reported by Gokaslan et al. on 400 patients who underwent transthoracic vertebrectomy, 3 patients experienced neurological deterioration following preoperative embolization of spinal metastatic tumor. ${ }^{8}$

Based on the above-cited controversy in the literature, the importance of the radicular arteries, particularly the AKA, for maintaining spinal cord blood flow and function is still unclear. ${ }^{1}$ In the present study we sought to check the correlation between EPM-controlled radicular vessel occlusion (temporary or permanent) and patients' clinical outcomes. Furthermore, we aimed to evaluate whether EPM during angiography and temporary vessel occlusion can guide the surgeon regarding which one of the radicular arteries can be sacrificed without causing untoward neurological complications.

The patients in our case series underwent extensive preoperative arterial occlusions. The AKA was closed in 3 patients, 3 bilateral radicular arteries were obliterated in 4 patients, and 4-level bilateral radicular arteries were occluded in an additional patient. It is noteworthy that a patent ASA was demonstrated following the endovascular occlusion in each of the patients. The EPM was not affected by temporary or permanent closure of the vessels in any of the cases, and furthermore, all of our patients managed to maintain their neurological status postoperatively. Of note, 2 of our patients suffered from wound infection and required revision surgery. However, we think that this complication is not related to embolization but rather to the extended length of the incision, invasiveness of the procedure, and long operative time.

Our study, although it was conducted in a limited group of patients, shows that a good correlation exists between the EPM and clinical outcome after an endovascular spinal procedure in which radicular arteries, including the AKA, were occluded. This observation suggests that EPM might be considered a useful tool in evaluating the vascular supply to the spinal cord during endovascular procedures. Furthermore, our data were in accordance with those of Boriani et al. ${ }^{3}$ and Murakami et al.; ${ }^{17}$ that closure of radicular arteries at 3 vertebral levels, including the AKA, can be well tolerated by the spinal cord, without untoward neurological sequelae.
A plausible explanation of the spinal cord's ability to sustain curtailment of the radicular artery blood supply may be found in the complex vascular anatomy, ${ }^{6}$ which includes 3 main arterial channels, a single ASA, and paired PSAs on the surface, as well as a meshlike pial plexus surrounding the spinal cord. This rich anastomotic network may offer an alternative route for arterial blood flow ${ }^{14}$ and serve as a collateral system at times of stress, such as vascular obstruction or cord compression. This pial vascular anatomy may also explain the immediate filling of the ASA after radicular artery occlusion, as was seen in our patients.

However, considering the previous reports on neurological deterioration secondary to occlusion of radicular arteries in the course of different surgical and endovascular procedures, the use of EPM during preoperative temporary endovascular closure of these vessels might be a useful tool to predict the safety of occlusion of the involved vessels.

\section{Conclusions}

The use of EPM coupled with spinal angiography and temporary arterial occlusion may guide decision making regarding the safety of permanent radicular artery occlusion, including the AKA, in patients undergoing TES for spine tumors. Our study comprises a small group of patients, and thus its conclusions should be treated with caution until a larger study reinforces these observations.

\section{References}

1. Apel DM, Marrero G, King J, Tolo VT, Bassett GS: Avoiding paraplegia during anterior spinal surgery. The role of somatosensory evoked potential monitoring with temporary occlusion of segmental spinal arteries. Spine (Phila Pa 1976) 16 (8 Suppl):S365-S370, 1991

2. Awad AW, Almefty KK, Ducruet AF, Turner JD, Theodore $\mathrm{N}$, McDougall CG, et al: The efficacy and risks of preoperative embolization of spinal tumors. J Neurointerv Surg [epub ahead of print], 2015

3. Boriani S, Bandiera S, Gasbarrini A, Corghil A, Simonetti $\mathrm{L}$ : The role of cord vascularization in planning spine oncologic surgery. ArgoSpine News J 18:47, 2008 
4. Charles YP, Barbe B, Beaujeux R, Boujan F, Steib JP: Relevance of the anatomical location of the Adamkiewicz artery in spine surgery. Surg Radiol Anat 33:3-9, 2011

5. Chi JH, Sciubba DM, Rhines LD, Gokaslan ZL: Surgery for primary vertebral tumors: en bloc versus intralesional resection. Neurosurg Clin N Am 19:111-117, 2008

6. Crock HV: The arterial supply and venous drainage of the vertebral column of the dog. J Anat 94:88-99, 1960

7. Dommisse GF: The blood supply of the spinal cord. A critical vascular zone in spinal surgery. J Bone Joint Surg Br 56:225-235, 1974

8. Gokaslan ZL, York JE, Walsh GL, McCutcheon IE, Lang FF, Putnam JB Jr, et al: Transthoracic vertebrectomy for metastatic spinal tumors. J Neurosurg 89:599-609, 1998

9. Gokaslan ZL, Zadnik PL, Sciubba DM, Germscheid N, Goodwin CR, Wolinsky JP, et al: Mobile spine chordoma: results of 166 patients from the AOSpine Knowledge Forum Tumor database. J Neurosurg Spine 24:644-651, 2016

10. Griepp RB, Ergin MA, Galla JD, Lansman S, Khan N, Quintana $\mathrm{C}$, et al: Looking for the artery of Adamkiewicz: a quest to minimize paraplegia after operations for aneurysms of the descending thoracic and thoracoabdominal aorta. J Thorac Cardiovasc Surg 112:1202-1215, 1996

11. Hsieh PC, Li KW, Sciubba DM, Suk I, Wolinsky JP, Gokaslan ZL: Posterior-only approach for total en bloc spondylectomy for malignant primary spinal neoplasms: anatomic considerations and operative nuances. Neurosurgery 65 (6 Suppl):173-181, 2009

12. Johnson JP, Pare LS, Torres RA: Thoracolumbar body replacement: material and techniques. Contemp Neurosurg 20:1-9, 1998

13. Kawahara N, Tomita K, Murakami H, Demura S: Total en bloc spondylectomy for spinal tumors: surgical techniques and related basic background. Orthop Clin North Am 40:47-63, vi, 2009

14. Lazorthes G, Gouaze A, Zadeh JO, Santini JJ, Lazorthes Y, Burdin P: Arterial vascularization of the spinal cord. Recent studies of the anastomotic substitution pathways. J Neurosurg 35:253-262, 1971

15. Matsuda H, Fukuda T, Iritani $O$, Nakazawa $T$, Tanaka $H$, Sasaki H, et al: Spinal cord injury is not negligible after TEVAR for lower descending aorta. Eur J Vasc Endovasc Surg 39:179-186, 2010

16. Matsuda H, Ogino H, Fukuda T, Iritani O, Sato S, Iba Y, et al: Multidisciplinary approach to prevent spinal cord ischemia after thoracic endovascular aneurysm repair for distal descending aorta. Ann Thorac Surg 90:561-565, 2010

17. Murakami H, Kawahara N, Tomita K, Demura S, Kato S, Yoshioka K: Does interruption of the artery of Adamkiewicz during total en bloc spondylectomy affect neurologic function? Spine (Phila Pa 1976) 35:E1187-E1192, 2010

18. Nishi H, Mitsuno M, Tanaka H, Ryomoto M, Fukui S, Miyamoto Y: Spinal cord injury in patients undergoing total arch replacement: a cautionary note for use of the long elephant technique. J Thorac Cardiovasc Surg 142:1084-1089, 2011

19. Orchowski J, Bridwell KH, Lenke LG: Neurological deficit from a purely vascular etiology after unilateral vessel ligation during anterior thoracolumbar fusion of the spine. Spine (Phila Pa 1976) 30:406-410, 2005

20. Oskouian RJ Jr, Johnson JP: Vascular complications in anterior thoracolumbar spinal reconstruction. J Neurosurg 96 (1 Suppl):1-5, 2002

21. Quraishi NA, Gokaslan ZL, Boriani S: The surgical management of metastatic epidural compression of the spinal cord. J Bone Joint Surg Br 92:1054-1060, 2010

22. Sciubba DM, Petteys RJ, Dekutoski MB, Fisher CG, Fehlings MG, Ondra SL, et al: Diagnosis and management of metastatic spine disease. A review. J Neurosurg Spine 13:94108,2010

23. Tomita K, Toribatake Y, Kawahara N, Ohnari H, Kose H: Total en bloc spondylectomy and circumspinal decompression for solitary spinal metastasis. Paraplegia 32:36-46, 1994

24. Turnbull IM: Microvasculature of the human spinal cord. J Neurosurg 35:141-147, 1971

25. Ueda Y, Kawahara N, Tomita K, Kobayashi T, Murakami H, Nambu K: Influence on spinal cord blood flow and function by interruption of bilateral segmental arteries at up to three levels: experimental study in dogs. Spine (Phila Pa 1976) 30:2239-2243, 2005

26. Villavicencio AT, Oskouian RJ, Roberson C, Stokes J, Park J, Shaffrey CI, et al: Thoracolumbar vertebral reconstruction after surgery for metastatic spinal tumors: long-term outcomes. Neurosurg Focus 19(3):E8, 2005

27. Yamazaki T, McLoughlin GS, Patel S, Rhines LD, Fourney DR: Feasibility and safety of en bloc resection for primary spine tumors: a systematic review by the Spine Oncology Study Group. Spine (Phila Pa 1976) 34 (22 Suppl):S31-S38, 2009

28. Yao KC, Boriani S, Gokaslan ZL, Sundaresan N: En bloc spondylectomy for spinal metastases: a review of techniques. Neurosurg Focus 15(5):E6, 2003

\section{Disclosures}

The authors report no conflict of interest concerning the materials or methods used in this study or the findings specified in this paper.

\section{Author Contributions}

Conception and design: Salame, Lidar. Acquisition of data: Salame, Maimon, Regev, Kimchi, Korn, Lidar. Drafting the article: Salame. Critically revising the article: Salame, Regev, Mangel. Reviewed submitted version of manuscript: all authors. Approved the final version of the manuscript on behalf of all authors: Salame. Administrative/technical/material support: Mangel. Study supervision: Salame.

\section{Correspondence}

Khalil Salame, Department of Neurosurgery, The Spine Unit, Tel Aviv Medical Center, 6 Weizmann St., 64239 Tel Aviv, Israel. email: salame@tasmc.health.gov.il. 